\title{
Linx
}

Revue des linguistes de l'université Paris X Nanterre

$80 \mid 2020$

L'héritage de Jean Dubois et Françoise Dubois-Charlier

\section{Explicitation de l'intersubjectivité et types de modalités}

The growing explicitness of intersubjectivity within various types of modality

\section{Olivier Polge}

\section{(2) OpenEdition \\ 12 Journals}

\section{Electronic version}

URL: http://journals.openedition.org/linx/6411

DOI: 10.4000/linx.6411

ISSN: 2118-9692

\section{Publisher}

Presses universitaires de Paris Nanterre

\section{Electronic reference}

Olivier Polge, «Explicitation de l'intersubjectivité et types de modalités », Linx [Online], 80 | 2020, Online since 10 July 2020, connection on 05 August 2020. URL : http://journals.openedition.org/linx/6411 ; DOI : https://doi.org/10.4000/linx.6411

This text was automatically generated on 5 August 2020

Département de Sciences du langage, Université Paris Ouest 


\section{Explicitation de l'intersubjectivité et types de modalités}

The growing explicitness of intersubjectivity within various types of modality

Olivier Polge

\section{Introduction}

Le présent article est le fruit d'un rapprochement au niveau de l'explicitation de l'intersubjectivité entre plusieurs études relevant de différents domaines: mon mémoire de maitrise portant sur la traductologie, une partie diachronique de ma thèse et enfin mon expérience en didactique de l'anglais en tant que responsable de Master MEEF. Françoise Dubois-Charlier a dirigé mon mémoire de maîtrise en 1995 sur la traduction des adverbes anglais en -ly vers le français. Ce travail avait pour point de départ une étude comparative des adjectifs et adverbes en anglais et en français présentée par Françoise Dubois-Charlier en cours de linguistique d'option de troisième année de Licence. Cette étude a donné lieu à la publication J. Dubois \& F. DuboisCharlier (2005) ${ }^{1}$.

L'article inspiré du mémoire de maitrise ${ }^{2}$ dégage des critères pour la transposition des adverbes anglais portant sur le sujet du verbe et exprimant souvent un état subjectif en préposition + nom en français. L'un d'entre eux est l'explicitation de la subjectivité du sujet par le narrateur-énonciateur, dans ses échanges verbaux ou interactions visuelles, par exemple au niveau du ton employé ou de la manière de regarder l'autre, ce rapport de force étant explicité par le narrateur-énonciateur.

Françoise Dubois-Charlier m'a ensuite préparé efficacement aux concours de recrutement du second degré. Ses cours de concours en syntaxe me sont régulièrement utiles, notamment sur les relatives et les inversions en anglais. Le polycopié de syntaxe qu'elle avait rédigé a donné lieu à la publication F. Dubois-Charlier \& B. Vautherin, (1997), qui reste un ouvrage de référence en syntaxe anglaise pour tout angliciste ${ }^{3}$. Françoise Dubois-Charlier a enfin participé à mon jury de thèse ${ }^{4}$. Cette thèse portait entre autres sur l'évolution du sens du prédicat want en diachronie du manque au désir. 
Cette évolution est due à l'explicitation graduelle de la demande implicite adressée par le locuteur à son interlocuteur. On retrouve donc en diachronie le phénomène d'explicitation du conflit de pouvoir entre les deux interlocuteurs ${ }^{5}$.

L'intersubjectivité prend la forme d'un conflit de pouvoir par lequel le locuteur vise à faire agir l'interlocuteur selon leur propre choix. E. Gilbert (1993: 93) définit la modalité intersubjective, ou modalité de rang 4, couvrant entre autres les sens d'ordre, de permission, de suggestion, d'interdiction, comme celle permettant à l'énonciateur d'influer sur autrui a priori, donc en amont de l'action visée, notamment sur le coénonciateur. Mais nous montrerons en $3 c$ que la modalité appréciative, ou modalité de rang 3, définie comme un jugement qualitatif a posteriori toujours par E. Gilbert (1993 : 93), vise elle aussi à exercer sur autrui une contrainte a priori dans ces contextes d'interaction.

L'étude qui suit établit des comparaisons entre différents domaines de l'anglistique que sont la traduction, la diachronie et la didactique au niveau de l'explicitation des relations intersubjectives. Il s'agira de déterminer pour chacun quelles voies l'explicitation emprunte, à quels moyens linguistiques elle recourt et jusqu'où elle va.

On trouve dans l'ouvrage de traductologie Vinay \& Darbelnet la définition suivante de l'explicitation:

«Procédé qui consiste à introduire dans LA [langue d'arrivée] des précisions qui restent implicites dans LD [langue de départ], mais qui se dégagent du contexte ou de la situation ». Vinay \& Darbelnet (1958, 1967: 9)

Le contexte mentionné par Vinay \& Darbelnet sera modélisé par un système de coordonnées spatio-temporelles et subjectives dans le cadre de la Théorie des Opérations Énonciatives d'Antoine Culioli. La formule de repérage, ou référentiel que donne A. Culioli (1990: 80) pour une lexis, notée $\lambda$, et qu'il appelle (1990:130) « cascade de sits ", est la suivante :

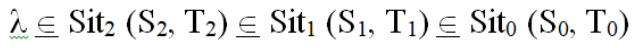

(repère de l'événement) (repère de l'acte de locution) (repère de l'énonciation origine)

Cette formule repose sur l'opération de repérage, notée $\underline{E}$. Dans le repérage $\mathrm{x} \in \mathrm{y}$, y est l'élément repère et x l'élément repéré.

Les paramètres qui constituent cette formule sont donc repérés les uns par rapport aux autres dans le cadre d'un système de coordonnées. La TOE ramène en effet l'activité de langage à une opération fondamentale de repérage. Antoine Culioli la définit de la façon suivante :

«L'observation minutieuse de langues variées et la théorisation de phénomènes en apparence éloignés, m'ont amené à poser une relation fondamentale appelée: relation de repérage, construite par l'opération élémentaire primitive dite opération de repérage. Le concept de repérage est lié au concept de localisation relative et à celui de détermination. Dire que $\mathrm{x}$ est repéré par rapport à y signifie que x est localisé (au sens abstrait du terme), situé par rapport à y, que ce dernier, qui sert de repère (point de référence) soit lui-même repéré par rapport à un autre repère, ou à un repère origine ou qu'il soit lui-même origine ». A. Culioli (1999 : 97).

En guise d'illustration de cette formule, on s'appuiera sur des passages d'œuvres littéraires, traduites ou enseignées, dans lesquelles est présent un énonciateurnarrateur assimilé au repère $S_{0}$. Celui-ci rapporte des échanges entre différents locuteurs qui s'identifient au repère $S_{1}$ dès lors qu'ils prennent la parole et repèrent leur interlocuteur $\mathrm{S}_{1}^{\prime}$ par rapport à eux par différenciation. 
Le domaine abordé en traduction illustre également la distinction entre situation de locution $\operatorname{Sit}_{1}\left(\mathrm{~S}_{1}, \mathrm{~T}_{1}\right)$ et situation d'énonciation $\mathrm{Sit}_{0}\left(\mathrm{~S}_{0}, \mathrm{~T}_{0}\right)$ dans le sens où le narrateurénonciateur $S_{0}$ explicite la relation entre locuteur $S_{1}$ et interlocuteur $S_{1}^{\prime}$.

En discours direct non rapporté, énonciateur et locuteur coïncident, et le repère $S_{1}$ est identifié à $\mathrm{S}_{0}$, si bien que la distinction entre situation de locution $\operatorname{Sit}_{1}\left(\mathrm{~S}_{1}, \mathrm{~T}_{1}\right)$ et situation d'énonciation $\mathrm{Sit}_{0}\left(\mathrm{~S}_{0}, \mathrm{~T}_{0}\right)$ est peu pertinente. C'est plutôt dans ce cadre que se situe l'intersubjectivité en diachronie.

En didactique, l'explicitation des contraintes intersubjectives peut aussi se faire à propos de procès relevant d'une situation d'événement $\operatorname{Sit}_{2}\left(\mathrm{~S}_{2}, \mathrm{~T}_{2}\right)$. L'explicitation qui en est faite est donc notamment d'ordre spatio-temporel $\left(\mathrm{T}_{2}\right)$ et subjectif $\left(\mathrm{S}_{2}\right)$.

Nous commencerons l'étude par l'explicitation de l'intersubjectivité en traduction.

\section{Explicitation de la relation interlocutoire dans les traductions d'Alice's Adventures in Wonderland en français}

\section{1a. Hyponymes adjectivaux et participes verbaux}

Le corpus à partir duquel l'explicitation des relations interlocutoires sera observé est constitué de plusieurs traductions d'Alice's Adventures in Wonderland. Les relations interpersonnelles y sont très présentes car l'héroïne rencontre toutes sortes de personnages qui lui posent des défis ou défendent âprement leur point de vue par rapport à cette intruse, ce qui rend le corpus pertinent pour l'étude des relations intersubjectives, notamment à l'occasion des rencontres entre les personnages. Plusieurs traductions d'Alice's Adventures in Wonderland sont comparées dans Polge (2013) pour mettre en lumière des phénomènes communs à des traducteurs différents pour la traduction des adverbes en -ly incidents au sujet du verbe :

(1) The mouse looked at her rather inquisitively,

(1a) La souris la regarda avec un brin de curiosité (Merle)

(1b) La Souris la regarda d'un air inquisiteur (Bué, Prophétie)

(1c) La Souris la regarda d'un air interrogateur (Lapalme)

Les adjectifs inquisiteur et interrogateur en (1b) et (1c) sont des hyponymes de curieux/ curiosité. Ces termes plus précis ajoutent donc une nuance. L'hypothèse émise est que ces hyponymes explicitent la relation intersubjective entre la souris et Alice. En effet, l'adjectif anglais inquisitive est défini par le Longman Dictionary of Contemporary English (1987) de la façon suivante : «trying to find out (too many) details about things and people ». L'adjectif français inquisiteur est quant à lui défini par le dictionnaire Le Robert 2019 dans les termes suivants: "Qui interroge indiscrètement ou de façon autoritaire ». Ces définitions soulignent le rapport de force entre le locuteur et son interlocuteur. Même si certains traducteurs décident de ne pas expliciter la relation intersubjective en traduisant par avec curiosité, les autres traducteurs explicitent l'antagonisme entre les personnages.

Les énoncés (2), (3) et (4) prennent pour sujet Alice. En (2), l'adverbe thoughtfully, censé renvoyer à un état subjectif inaccessible que pourrait rendre l'adjectif pensif en français, est traduit par le groupe prépositionnel avec prudence, dans lequel le nom prudence révèle le caractère dangereux du contexte interlocutoire pour Alice. En effet, 
son interlocuteur n'hésite pas à l'interrompre et la contredire systématiquement dans une conversation pleine de quiproquos. La traduction de cet adverbe explicite donc la relation que le référent du sujet entretient avec son interlocuteur :

(2) «Thank you, it's a very interesting dance to watch », said Alice, feeling very glad that it was over at last: « and I do so like that curious song about the whiting! »

"Oh, as to the whiting, " said the Mock Turtle, "they-you've seen them, of course?"

"Yes, said Alice, "I've often seen them at dinn-» she checked herself hastily.

"I don't know where Dinn may be, " said the Mock Turtle, "but if you've seen them so often,

of course you know what they're like."

"I believe so," Alice replied thoughtfully. "They have their tails in their mouths-and

they're all over crumbs."

"You're wrong about the crumbs," said the Mock. Turtle (...).

(2a) - Il me semble, répondit Alice avec prudence. (Merle)

En (3), cautiously est rendu par une traduction contenant une nominalisation du verbe ménager, verbe transitif qui implique un deuxième argument correspondant à l'interlocuteur :

(3) Alice did not wish to offend the Dormouse again, so she began very cautiously:

(3a) Soucieuse de ne pas froisser le Loir de nouveau, Alice revint à la charge avec

beaucoup de ménagements (Merle)

En (4), softly est traduit par des participes présents caressante et suppliante en (4c) et (4e). Ces participes sont également issus de verbes transitifs directs qui impliquent un deuxième argument correspondant à l'interlocuteur :

(4) So she called softly after it, "Mouse dear! Do come back again

(4a) Elle l'appela donc d'une voix douce (Rouard)

(4b) Alice l'appela donc d'une voix doucereuse (Parisot)

(4c) Alice l'appela d'une voix caressante (Bay, Marcireau)

(4d) Elle le supplia de revenir, lui dit mille choses douces (Scott - Keukles)

(4e) Pour l'apaiser, Alice l'appela d'une voix suppliante (Peter)

(4f) Alice l'appela de sa plus douce voix (Lapalme)

Les variations qu'effectuent les autres traducteurs autour du mot doux dans les traductions (4b), (4d), (4f), par le biais de suffixes tels que -euse dans doucereuse, de superlatifs tels que sa plus douce voix ou d'adjectifs numéraux tels que mille, renvoyant à un nombre élevé, explicitent l'effort de politesse que consent Alice vis-à-vis de la souris par l'apport de déterminations supplémentaires. Cet apport de déterminations est permis par l'emploi du nom support voix. Ces noms supports (air, oeil, voix, pas) sont souvent la trace d'un raisonnement d'inférence du narrateur qui tente de déterminer l'intention du personnage (cf. Polge, $2013: 165$ ).

Dans trois traductions de (5), angrily est rendu par le nom support air suivi des participes passés outragé et offensé, qui eux aussi sont issus de verbes transitifs :

(5) «It is a very good height indeed! » said the Caterpillar angrily, rearing itself upright as it spoke.

(5a) C'est une taille très convenable, au contraire ", riposta, en se redressant de toute sa hauteur et en prenant un air outragé, le Bombyx. (Parisot)

(5b) - Mais c'est une taille très raisonnable! rétorqua le Ver à soie en se redressant de toute sa hauteur et en prenant un air offensé (...) (Lattès, Rouard)

La présence de verbes transitifs dans les traductions de ces exemples nous amène à proposer une modélisation qui rende compte des arguments de ces verbes, lesquels explicitent les participants au procès, avant de nous intéresser aux autres aspects de ces traductions. 


\section{1b. Modélisation}

L'explicitation de la relation entre locuteur $\mathrm{S}_{1}$ et co-locuteur $\mathrm{S}_{1}{ }_{1}$ implique un schéma prédicatif $<\mathrm{a} \mathrm{r} b$ incluant un verbe ou prédicat $r$ dont le terme $a$ est identifié à $\mathrm{S}_{1}$ pour les traductions des exemples (3) et (4). Dans les traductions de (5), les participes passés outragé et offensé impliquent que c'est le terme $b$ qui est thématisé et identifié à $\mathrm{S}_{1}$. La relation entre locuteur $S_{1}$ et interlocuteur $S^{\prime}{ }_{1} n^{\prime}$ est toutefois pas totalement explicitée puisque l'argument a du prédicat, qui prendrait la forme d'un complément d'agent, n'est pas mentionné. Dans tous les cas, cette amorce d'explicitation dans le processus de traduction est attribuée à l'énonciateur-narrateur qui rapporte les dialogues et prend en charge les échanges. Le schéma prédicatif est donc repéré par rapport au paramètre $S_{0}$ dans la «cascade de sits " dans le schéma suivant où $a$ apparaît entre parenthèses, puisqu'il n'est pas explicité dans les traductions de (5). Par contre, $b$ est thématisé et identifié à $\mathrm{S}_{1}$ :

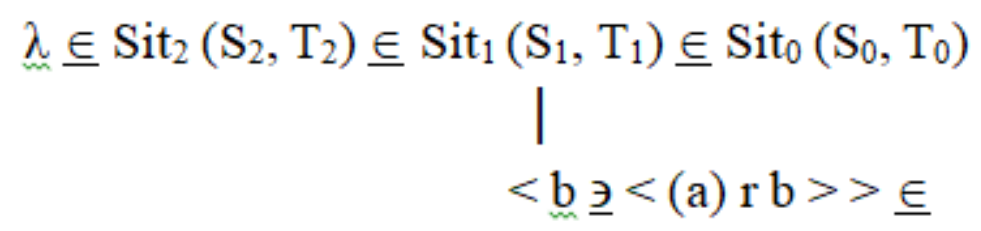

Suite à cette modélisation, on peut se demander si cette explicitation de la relation interlocutoire correspond à l'explicitation des relations telle que l'entendent certains auteurs à propos de l'anglais, notamment J. Guillemin-Flescher (1981).

\section{1c. Différences avec l'explicitation des relations prédicatives en anglais}

L'explicitation des relations prédicatives se vérifie en anglais, comme le montre $\mathrm{J}$. Guillemin-Flescher (1981 : 108) à travers des traductions d'Emma Bovary vers l'anglais. Elle y remarque l'emploi de relateurs tels que les déterminants possessifs, with et les participes présents. Les déterminants possessifs explicitent les relations entre les groupes d'une même proposition, par exemple le sujet et le complément, en d'autres termes les arguments $a$ et $b$ du schéma prédicatif $<a \mathrm{r} \mathrm{b}>$ présent dans ces propositions. Les participes présents et la conjonction with constituent ou introduisent un verbe subordonné au verbe principal conjugué et les premiers arguments des deux verbes sont identifiés :

(6) Quand il arrivait à la distribution des récompenses, il dépeignait la joie des lauréats en traits dithyrambiques. "Le père embrassait son fils, le frère le frère, l'épouse l'époux".

(6a) Fathers embraced their sons, brothers brothers, husbands wives. (Hopkins)

(6b) Fathers embraced their sons, brothers their brothers, husbands their wives. (May)

(7) Deux vieilles femmes, de larges cabas de toile cirée à la main, discutaient à voix haute des mérites comparés de l'huile Dulcine et de la margarine Planta.

(7a) Two old women carrying broad oilcloth shopping bags were loudly debating the relative merits of Dulcine oil and Planta margarine.

(8) Le pharmacien s'éloigna d'un pas rapide, sourire aux lèvres et jarret tendu.

(8a) The chemist made off with a smile on his lips, at a dazzling stride.

(9) Eux se cognaient pour de bon, dents serrées, visages en sueur

(9a) They fought in earnest, clenching their teeth, with sweat pouring down their faces. 
Toutefois, ces relations, établies au niveau prédicatif ( $\lambda$ dans la cascade de sits, remplaçable par $<\mathrm{ar} b>$ ) ne correspondent pas au cas que nous avons abordé en $1 \mathrm{~b}$ qui est celui de l'explicitation de la relation interlocutoire, relevant du niveau énonciatif car impliquant une identification à des paramètres du référentiel énonciatif (plus précisément le repère de l'acte de locution $\operatorname{Sit}_{1}\left(\mathrm{~S}_{1}, \mathrm{~T}_{1}\right)$ ).

\section{1d. Explicitation de la modalité appréciative exprimée et communication émotive}

En impliquant dans les traductions de (5) un complément d'agent non mentionné qui correspondrait à l'interlocuteur, ici Alice, ainsi qu'un changement d'état du sujet de l'énoncé, les participes passés ou passifs outragé et offensé explicitent la modalité appréciative exprimée par l'adjectif angry:

(5) «Well, I should like to be a little larger, sir, if you wouldn't mind, " said Alice: «three inches is such a wretched height to be".

"It is a very good height indeed!» said the Caterpillar angrily, rearing itself upright as it spoke. (it was exactly three inches high).

«But I'm not used to it! » pleaded poor Alice in a piteous tone. And she thought to herself, «I wish the creatures wouldn't be so easily offended!»

(5a) C'est une taille très convenable, » au contraire, riposta, en se redressant de toute sa hauteur et en prenant un air outragé, le Bombyx. (Parisot)

(5b) - Mais c'est une taille très raisonnable! rétorqua le Ver à soie en se redressant de toute sa hauteur et en prenant un air offensé (...) (Lattès, Rouard)

La modalité appréciative est définie par Gilbert (1993: 93) comme un jugement qualitatif a posteriori de l'énonciateur-narrateur. Selon Simonin (2015), «lorsque la modalisation est donnée a posteriori, locuteur et allocutaire partagent pour connaissance (ou le locuteur fait comme si c'était le cas) le caractère avéré ou contrefactuel du procès ».

Par le biais du narrateur et des traducteurs ayant recours à ces participes passés, la chenille est perçue comme étant affectée par un changement d'état subjectif irréversible causé par son interlocutrice.

Si l'on se concentre toujours sur les mêmes traductions de (5), on note également la présence des gérondifs en prenant, qui indiquent que l'air pris par le personnage est intentionnel et simulé. Cette simulation constitue une forme de communication émotive, que Caffi et Janney (1994: 327), à la suite de Marty (1908), définissent comme le produit d'une élaboration secondaire, non spontanée, dans le but de faire agir autrui selon son propre choix dans le cadre d'un conflit de pouvoir :

emotive communication: the intentional strategic signalling of affective information in speech and writing (e. g. evaluative dispositions, evidential commitments, volitional stances, relational orientations, degrees of emphasis, etc.) in order to influence partner's interpretation of situations and reach different goals. (http://www.icar.cnrs.fr/membre/cplantin/emotions/)

On note dans la définition ci-dessus les adjectifs «intentional» (intentionnel), «strategic " (stratégique) et les expressions " relational orientations » (orientations relationnelles), « reach different goals » (atteindre des buts différents), ce qui rappelle l'idée d'un conflit de pouvoir au sein de la relation interlocutoire. La traduction explicite donc le caractère émotif de la réaction appréciative de la chenille dans le but de manipuler Alice. Cela nous amène à remettre en question la dimension a posteriori de 
cette modalité qui n'a d'autre but que d'exercer une contrainte a priori sur l'interlocuteur.

Si l'on essaie de trouver des structures grammaticales anglaises qui permettent elles aussi la manipulation de l'interlocuteur, on peut s'intéresser aux constructions want someone to et need someone to qui expriment une contrainte intersubjective en anglais actuel, mais diffèrent toutefois par le fait qu'ils expriment cette fois une modalité exclusivement a priori, située avant la réalisation de l'action visée. Or, ces structures exprimaient au départ un simple besoin. Les contextes propices à l'explicitation de leur sens de contrainte sont toutefois là encore des contextes interlocutoires. Même si cette évolution du besoin au désir et à la contrainte intersubjective pourrait être rendue en traduction, l'objectif de la partie qui suit est de mettre en évidence dans un autre domaine qu'est la diachronie l'expression d'un changement d'état dont la détermination du déclencheur s'est accrue au fil du temps.

\section{Explicitation de la relation interlocutoire en diachronie}

\section{2a. Du besoin à la contrainte intersubjective}

Les prédicats want et need ont développé des constructions N1 V1 N2 to V2, orientées vers un changement d'état. Dans leurs premiers emplois, N2 était peu déterminé (cf. Polge, 2015) pour les raisons suivantes: dans la construction N1 want N2 to V2 en particulier, N2 n'était pas forcément premier argument de V2 et renvoyait à de l'inanimé. En (10), pikes est COD de set :

(10) He wanted pikes to set before his archers (W. Shakespeare, 1 Henry 6, I. i. 116, 1589)

Même en cas de N2 renvoyant à de l'animé humain, celui-ci pouvait être deuxième argument de want, comme en (11), où talk régit une préposition isolée dont le complément ne peut être que someone. Someone ne peut donc pas être premier argument de talk:

(11) He was lonely and wanted someone to talk to. (A. Christie, The ABC Murders, p.

189, Fontana, 1936)

Cette indétermination a ensuite laissé place à une détermination croissante pour N2 qui a alors plus systématiquement renvoyé à de l'animé humain. Cette évolution s'est accompagnée d'un changement de fonction syntaxique pour ce deuxième argument: dès lors que N2 renvoie à de l'animé humain, on observe dans la majorité des exemples un $\mathrm{N} 2$ sujet de V2:

(12)

Lazarotto.

... I haue mischiefe

Within my breast, more then my bulke can hold:

I want a midwiue to deliuer it.

Lorenzo.

Ile be the hee one then, and rid thee soone

of this dull, leaden, and tormenting elfe ${ }^{6}$.

(T. Kyd, Ieronimo, 208 [I. iii. 8], 1605, cité par M. Berstchinger [1941])

(13)

"'The modern young man," said Aunt Dahlia, "is a congenital idiot and wants a nurse to lead him by the hand and some strong attendant to kick him regularly 
at intervals of a quarter of an hour." (P. G. Wodehouse, Very Good, Jeeves, p. 78, QPD, 1930)

Ces exemples comportent un N2 renvoyant à un animé humain, mais qui reste indéterminé, du fait de la présence de l'article indéfini a. L'exemple (13) a un sens générique et n'est pas plongé dans un contexte intersubjectif où the modern young man exercerait une contrainte sur autrui. Par contre, l'exemple (12) s'inscrit quant à lui dans du discours direct et à travers sa réponse, Lorenzo accepte de pallier le besoin exprimé par Lazarotto. La tirade de Lazarotto constitue donc une demande implicite.

A partir de la fin du XIX è siècle, N2 en est venu à désigner le co-locuteur à la deuxième personne, notamment dans le cadre du discours direct, comme dans les exemples (14) à (16). Cette évolution a explicité l'expression par want et need d'un désir, ou appréciation favorable explicite orientée vers la réalisation du procès désigné par V2, et donc d'une modalité intersubjective ayant pour cible N2 renvoyant à l'interlocuteur, désigné par you :

(14) I want for you and me to see everything as we seed it from fust to last. (WattsDunston, Aylwin xiv ch.2, 1898, cité par F. T. Visser (1963-1973 : III2, 2248)

(15) "Daydreaming as usual. (...) I need you to go into the village and fetch some food for me."

"Yes, Mother, I'll go at once," (...). (J. Archer, A Quiver full of Arrows, p. 60, Penguin, 1980)

(16) "I don't know nothin' an' I need for you to teach me." "You mean - you'll wait for me?" "Wait? Why, I'd wait fifty years for a chance - a chance to - a chance to better our stock and build better beef." (R. Condon, A Talent for Loving: or, The Great Cowboy Race, p.131, McGraw-Hill, 1961)

Cette explicitation a conféré à N2 le rôle le plus fréquent de sujet de V2 dans ces constructions, indiqué par l'emploi de for en (14) et (16) pour introduire N2, comme l'indiquent R. Quirk et al. (1985: 1193) :

For in Present-Day (mainly American) English "marks [the noun phrase] as the subject of an infinitive clause, rather than as object of the main clause"

F. T. Visser (1963-1973 : III2, 2244) précise que l'apparition de for après want date de la fin du XIX ${ }^{\text {ème }}$ siècle et constitue donc un phénomène récent :

"Apart from a few exceptions (...), the idiom is hardly ever met with before the

beginning of the twentieth century or the last years of the nineteenth century."

Il reste à expliquer le passage pour ces prédicats d'une demande implicite, n'identifiant pas sa cible, à un ordre explicite, identifiant sa cible et explicitant le sens de la contrainte exercée : la modalité intersubjective, ou radicale consiste pour un locuteur à demander à son interlocuteur d'agir selon son choix. Pour qu'elle soit efficace, la contrainte exprimée doit être présentée de façon implicite à l'interlocuteur comme un besoin non intentionnel et imposé par la situation. Néanmoins, au fil du temps, le procédé devient évident jusqu'à ce que les termes utilisés, qui expriment une volonté explicite du locuteur, nécessitent un remplaçant qui exprime une contrainte situationnelle. En conséquence, les conditions d'emploi des verbes au niveau énonciatif ont modifié le faisceau de relations dont ils sont la trace ${ }^{7}$.

Comme en partie $1 \mathrm{~d}$ où il était question de communication émotive, on note à propos de want et need des décalages entre le sens littéral d'un énoncé et l'intention réelle de son énonciateur grâce au contexte dans le cadre de l'expression de la modalité intersubjective. Ces décalages concernent également les auxiliaires modaux de l'anglais, qui eux aussi expriment une modalité intersubjective. 


\section{2b. Evolution du système modal de l'anglais}

M.-L. Groussier (1993) met ces décalages en évidence dans l'histoire de marqueurs de contrainte intersubjective appartenant à plusieurs langues indo-européennes. M.-L. Groussier (2000 et 2002) estime qu'ils donnent lieu à un glissement de sens, qui est à l'origine du renouvellement des systèmes de modalité intersubjective de quatre langues indo-européennes, dont l'anglais et le français. Ce renouvellement du système modal de l'anglais a été schématisé dans le tableau 1, où l'on observe un remplacement de l'auxiliaire modal shall par must, puis par have to.

Tableau 1

\begin{tabular}{|l|l|}
\hline De l'expression d'une contrainte non subjective & You shall go now. \\
\hline $\begin{array}{l}\text { Sculan (OE), debere (latin) = devoir de l'argent } \\
\text { (contrainte imposée par la société) }\end{array}$ & Yu dois partir. \\
\hline $\begin{array}{l}\text { Remplacement de shall } \\
\text { par must ('il y a nécessité' en vieil anglais) }\end{array}$ & $\begin{array}{l}\text { Expression par have to d'un ordre en } \\
\text { Remplacement de devoir par } \text { il faut, de } \\
\text { must par have to : I have to go. }\end{array}$ \\
\hline
\end{tabular}

On a également assisté à plusieurs remplacements successifs de l'auxiliaire will dont l'étymologie est la même que vouloir en français :

1) par shall à la première personne, puis par want to (“j'ai besoin”) ;

2) want est à son tour remplacé par I would like to et want au prétérit, ou need: I wanted to speak to you, I need to speak to you.

Want, qui exprimait le manque, a été utilisé comme procédé d'atténuation du désir de l'énonciateur et/ou du sujet de l'énoncé, jusqu'à ce que le procédé devienne évident et que d'autres formes atténuées (need, would like) viennent le remplacer. Want et need ont observé ce glissement de repérages situationnels, impliquant les paramètres $T_{1-0} d u$ référentiel, puisqu'ils exprimaient un besoin situationnel, vers des repérages subjectifs impliquant les paramètres $\mathrm{S}_{1-0}$ du référentiel.

M.-L. Groussier (1993) définit cette explicitation des paramètres subjectifs comme un phénomène de subjectivisation, ou expression de plus en plus claire du point de vue d'un animé humain au fil du temps, à la suite de E. C. Traugott (1995: 32 et 2010: 60) :

"Subjectification in grammaticalization' is, broadly speaking, the development of a grammatically identifiable expression of speaker belief or speaker attitude to what is said."

"Subjectification is the development of meanings that express speaker attitude or viewpoint (...)"

Alors que la relation interlocutoire est passée sous silence au départ par un procédé d'atténuation qui consiste à demander quelque chose en le présentant comme un besoin émanant de la situation, c'est cette même relation interlocutoire qui est donc à l'origine de la subjectivisation graduelle de ces mêmes marqueurs et donc de leur tombée en désuétude.

En anglais comme en français, des verbes qui expriment à l'origine un procès situationnel peuvent développer un autre emploi plus subjectif : alors que les verbes blesser et toucher expriment un procès situationnel, ces verbes peuvent exprimer un état subjectif et une relation intersubjective à partir du moment où l'un des deux arguments renvoie à l'énonciateur, comme dans ta lettre m'a touché / blessé ou j'ai été touché par ta 
lettre, et où l'autre argument renvoie à un élément situationnel qui confère au verbe un sens figuré. Annihilated, devastated, hurt, touched, moved désignent à l'origine :

- soit des catastrophes naturelles ou une forme de destruction ;

- soit des accidents ou événements corporels (cf. Polge, 2017).

Ce deuxième sens possible n'élude toutefois pas le sens de départ de ces verbes. On retrouve ces verbes à la forme participe passé, comme dans les traductions d'Alice en partie 1a, pour exprimer une modalité a posteriori.

De façon générale, l'explicitation de l'état subjectif de l'énonciateur est notamment due au passage d'un état de société où la subjectivité était occultée à une société émancipée. Or, en classe d'anglais, notamment dans le second degré, les élèves sont justement amenés à expliciter l'avis des personnages dans des œuvres étudiées datant d'il y a quelques siècles. Les textes étudiés concernent également des sociétés exerçant des contraintes sur leurs sujets.

Le volet didactique de cette étude est donc dans le prolongement de l'analyse de verbes orientés vers l'accomplissement d'une action étudiés en partie 2 .

Dans un cadre didactique, nous verrons en $3 c$ comment les emplois subjectifs de ces participes passés deviennent des outils de positionnement utiles à l'élève vis-à-vis d'un interlocuteur en interaction orale ou écrite et comment ces outils de communication émotive constituent des éléments de persuasion plus efficaces que le simple désaccord.

\section{Explicitation par les élèves dans un cadre didactique}

En classe d'anglais, la situation d'énonciation comprend bien sûr l'enseignant et surtout l'élève, qui est amené à expliciter les relations de pouvoir entre personnages et/ou interlocuteurs présents dans un texte littéraire à commenter, avec ou sans dialogue.

\section{3a. Explicitation de demandes implicites}

Les relations de pouvoir implicites étudiées en partie 2a sont notamment présentes dans les échanges théâtraux, illustrés par (11). Or, les élèves de cycle terminal étudient des extraits d'œuvres passées. Notamment dans le cadre de l'axe Art et pouvoir, sont étudiées des passages de tragédies de Shakespeare où le pouvoir est un enjeu: des personnages sont manipulés par d'autres, qui plus est dans une langue qui n'est pas l'anglais contemporain et qui nécessite d'être explicitée pour être comprise. Il s'agit donc de déterminer comment les élèves peuvent reconnaitre puis expliciter ces jeux de manipulation. On abordera la deuxième question avant de remonter vers la première à travers un dossier d'Epreuve de Mise en Situation Professionnelle du CAPES externe, qui contient des textes que les candidats au concours doivent didactiser.

Le dossier EMSP 33 du CAPES externe 2017 comporte un passage de MacBeth (acte I scène 7) dans lequel Lady Macbeth commandite l'assassinat de Duncan. Elle en fait à son mari la suggestion purement implicite en faisant avant tout appel à son estime de soi et à l'amour qu'elle lui porte. Cette demande doit être explicitée par les élèves afin qu'ils cernent les relations de pouvoir au sein du couple. Dans la pièce, le véritable cerveau est donc Lady MacBeth. Dans l'autre document du dossier à didactiser, la 
relation de pouvoir, cette fois entre Lady Anne et Richard III, est en sens inverse. Les relations conjugales en lien avec la notion de pouvoir à la Renaissance étaient donc au centre du dossier. On note dans le passage de MacBeth ci-dessous la recrudescence de formes modales à sens radical et dynamique utiles à réactiver pour les élèves, par exemple would de volition, qui ne vérifie plus exactement les mêmes emplois en anglais contemporain, puisqu'il se limite désormais au bon vouloir, et dare/durst (not) dans la bouche de Lady Macbeth qui mène entre guillemets une réflexion d'ordre métalinguistique :

MACBETH.

We will proceed no further in this business:

He hath honour'd me of late; and I have bought

Golden opinions from all sorts of people,

Which would be worn now in their newest gloss,

Not cast aside so soon.

LADY MACBETH.

Was the hope drunk

Wherein you dress'd yourself? Hath it slept since?

And wakes it now, to look so green and pale

At what it did so freely? From this time

Such I account thy love. Art thou afeard

To be the same in thine own act and valor

As thou art in desire? Wouldst thou have that

Which thou esteem'st the ornament of life,

And live a coward in thine own esteem;

Letting "I dare not" wait upon "I would,"

Like the poor cat $i$ ' the adage?

MACBETH.

Prythee, peace! I dare do all that may become a man; Who dares do more is none.

LADY MACBETH.

What beast was't, then,

That made you break this enterprise to me?

When you durst do it, then you were a man;

And, to be more than what you were, you would

Be so much more the man.

(W. Shakespeare, MacBeth, Act I scene 7, 1605)

L'apprenant est donc amené à expliciter la contrainte intersubjective exercée par Lady MacBeth sur son mari pour la mettre en perspective de façon critique. Cette contrainte va être explicitée dans une forme de discours narrativisé impliquant un verbe recteur à sens interlocutoire exprimant une demande, tel que ask, challenge, dare, defy, ou want. On quitte donc le discours direct à cette occasion. La reformulation :

(17a) She dares him to assassinate Duncan.

peut facilement être amenée par un énoncé intermédiaire contenant un verbe plus prototypique tel que want et donc facilement produit par les élèves, pour qu'ensuite les formes modales du texte de départ soient réutilisés avec une modification de leur faisceau de relations.

L'explicitation passe donc par l'apparition d'une relation prédicative enchâssante ; on passe du schéma (1) au schéma (2) : 
(1) $<$ a r b $\gg$ E $\operatorname{Sit}_{2}\left(S_{2}, T_{2}\right) \underline{E} \operatorname{Sit}_{1-0}\left(S_{1-0}, T_{1-0}\right)$

$$
(2) \ll \text { A R B }>\underline{\jmath}<\text { (a) r b } \gg \underline{\in} \operatorname{Sit}_{2}\left(S_{2}, T_{2}\right) \underline{E} \operatorname{Sit}_{1}\left(S_{1}, T_{1}\right) \underline{E} \operatorname{Sit}_{0}\left(S_{0}, T_{0}\right)
$$

En (1), le schéma prédicatif < a r b > désigne l'événement envisagé, par exemple «You shall kill Duncan ». En (2), le terme A, premier argument de l'enchâssante désigne l'origine subjective de la contrainte, ici Lady MacBeth et le terme $\mathrm{B}$, deuxième argument, constitue la cible de la contrainte qui accomplit le procès désigné par l'enchâssée $\langle$ (a) $r b>$, ici MacBeth. Un prédicat $R$ tel que defy explicite la contrainte exercée.

$\mathrm{Vu}$ que le texte contient des formes modales radicales, la reconnaissance des demandes implicites peut être menée à bien en faisant varier l'origine modale $\mathrm{A}$ en partant de la plus évidente, à savoir MacBeth, qui ne veut pas, n'ose pas passer à l'acte, pour ensuite en venir à Lady MacBeth, en posant aux élèves des questions du type «What does MacBeth want? " puis « What does Lady McBeth want? ».

Les relations entre personnages peuvent relever de degrés différents : elles sont soit de l'ordre de la demande, qui doit être implicite en vue d'une meilleure efficacité, soit de la contrainte, sans choix possible. Ces contraintes peuvent émaner de situations d'événements.

\section{3b. Explicitation de contraintes intersubjectives par le passif}

En compréhension écrite notamment, l'enseignant amène les apprenants à expliciter des événements subis par des populations, ou des abus de pouvoir s'étant produits dans des pays anglophones, par exemple le refoulement des Indiens d'Amérique du Nord, la condition de la génération perdue en Australie ou encore les Troubles en Irlande du Nord. Lors de la phase de compréhension, les énonciateurs apprenants reformulent des passages des textes supports afin d'expliciter les acteurs, le lieu et la période ainsi que les raisons de ces conflits de pouvoir pour mieux situer les événements, mais aussi accomplir des tâches visant à la réutilisation des structures manipulées. Le texte support étant le matériau de départ, sa reformulation est un moyen important permettant d'expliciter les événements. Le Bot et al. (2008:11) considèrent par ailleurs que la reformulation «se manifeste tout particulièrement dans les discours scientifiques, médiatiques et didactiques ». Ces discours peuvent s'entrecroiser en classe de langue vivante, comme nous le montrerons à travers les tâches proposées à la fin de cette sous-partie.

A titre d'exemple, (18) fait partie du dossier de EMSP 28 de la session 2017. Il est tiré du roman de Column McCann, Transatlantic, inspiré de la vie du célèbre esclave noir américain émancipé Frederick Douglass. Celui-ci se rend en Irlande en 1845, donc avant la Guerre de Sécession, pour promouvoir l'abolition de l'esclavage aux Etats Unis dans ses discours :

(18)

He was called upon to give a speech: his days as a slave, how he slept on a dirt floor in a hovel, crawled into a meal bag to stave off the cold, put his feet in the ashes for warmth. How he had lived with his grandmother for a while and had gone, then, to a plantation. Was taught, against the law, to read, write and spell. How he read the New Testament to his fellow slaves. Worked in a shipyard with Irishmen as companions. Ran 
away three times. Failed twice. Escaped Maryland at twenty years of age. (Column McCann, Transatlantic, New York: Random House, 2013)

Pour s'assurer de l'accès au sens du texte par des élèves de lycée, ceux-ci gagneraient à reformuler les verbes intransitifs utilisés dans le texte de départ par des verbes transitifs exprimant un changement d'état et impliquant davantage d'arguments. Le faisceau de relations qu'impliquent les prédicats utilisés sont donc pertinents en compréhension. On peut envisager la trace écrite suivante :

(18a)

He was asked to give a speech about his life as a slave.

He was separated from his grandmother and forced to go to a plantation because

he was certainly bought by another white man.

However, he was allowed to learn to read, write and spell by some of his new masters.

He was caught twice as he tried to escape.

He was annihilated when he failed to escape.

The audience was appalled by the slaves' living conditions.

Ces reformulations appellent des formes passives dont le complément d'agent n'est pas toujours explicité. Malgré cela, les verbes utilisés explicitent une relation intersubjective, par exemple une demande, avec ask, une autorisation, avec allow, ou une contrainte intersubjective, avec force ou make ou want ${ }^{8}$. Grâce au passif, déjà présent dans was called upon et was taught, que l'enseignant inviterait à imiter, les apprenants comprendraient les contraintes subies par un esclave à cette époque, mais aussi la chance qu'il a eue en tant qu'esclave de pouvoir apprendre à lire et à écrire, ce qui lui a permis ultérieurement de défendre sa cause.

Lors de l'étude de documents en compréhension, l'enseignant amène donc les apprenants à établir dans un premier temps des repérages d'ordre situationnel, concernant la situation matérielle des personnages (par exemple « He was separated from his grandmother" en (18a)), notamment par le biais de documents supplémentaires, par exemple des représentations iconographiques des ventes sur les marchés aux esclaves à l'époque.

Dans un deuxième temps, les apprenants établissent des relations d'ordre intersubjectif qui visent à déterminer les relations avec d'autres personnages (par exemple « He was forced to go to a plantation » en (18a)).

L'explicitation passe donc par l'apparition d'une relation prédicative enchâssante ; on passe d'un schéma (1) au schéma (2) :

$(1)<$ a r b $>\underline{E} \operatorname{Sit}_{2}\left(S_{2}, T_{2}\right) \underline{E} \operatorname{Sit}_{1}\left(S_{1}, T_{1}\right) \underline{E} \operatorname{Sit}_{0}\left(S_{0}, T_{0}\right)$

$(2) \ll$ A R B $>\underline{\jmath}<$ (a) r b $\gg \underline{\in} \operatorname{Sit}_{2}\left(\mathrm{~S}_{2}, \mathrm{~T}_{2}\right) \underline{\in} \operatorname{Sit}_{1}\left(\mathrm{~S}_{1}, \mathrm{~T}_{1}\right) \underline{\in} \mathrm{Sit}_{0}\left(\mathrm{~S}_{0}, \mathrm{~T}_{0}\right)$

En (1), le schéma prédicatif < a r b > désigne l'événement ayant eu lieu, par exemple « go to a plantation ». En (2), le terme A, premier argument de l'enchâssante désigne l'origine subjective de la contrainte, et le terme $\mathrm{B}$, deuxième argument, constitue la cible de la contrainte qui accomplit le procès désigné par l'enchâssée $<(a) r b\rangle$. Un prédicat $\mathrm{R}$ tel que force explicite la contrainte. Au passif, B est thématisé :

(3) $<$ B $\underline{\jmath}<<$ (A) R B $>\underline{\jmath}<$ (a) $\mathrm{r} b \gg>\underline{\epsilon} \operatorname{Sit}_{2}\left(\mathrm{~S}_{2}, \mathrm{~T}_{2}\right) \underline{\in} \operatorname{Sit}_{1}\left(\mathrm{~S}_{1}, \mathrm{~T}_{1}\right) \underline{\in} \operatorname{Sit}_{0}\left(\mathrm{~S}_{0}, \mathrm{~T}_{0}\right)$

Les structures causatives utilisées en compréhension peuvent ensuite être réinvesties en production: dans la mise en œuvre de leur compétence pragmatique, les élèves 
commentent des événements, marquant leur désaccord avec ces événements ou une opinion déclarée et persuadant autrui, tout en précisant les circonstances et la nature des événements, comme le ferait un journaliste. Une tâche possible consisterait donc à écrire un article rédigé par un journaliste de l'époque qui rapporterait ces conférences données par l'esclave émancipé tout en exprimant une réaction appréciative, comme en partie 1. Aussi bien en compréhension qu'en production, l'explicitation des rapports de force va de pair avec un jugement appréciatif a posteriori.

\section{3c. Explicitation des sentiments des personnages par le passif}

Lors de cette tâche, les sentiments de Frederick Douglass seraient explicités (par exemple grâce à « He was annihilated when he failed to escape » en (18a)). Suite à la partie $1 \mathrm{a}$, on peut affirmer que mentionner les sentiments des personnages permet de déterminer les relations qu'ils entretiennent entre eux, notamment si elles sont problématiques. C'est un début d'accès au sens et un début de conceptualisation. Or, il se trouve que le passif exprime souvent les sentiments en anglais (cf. Polge, 2017), mais aussi en français, comme le montre l'emploi des participes passifs dans la traduction des adverbes en (3). Cela est dû au fait que l'animé humain siège de ces sentiments est souvent thématisé, donc placé en position sujet.

L'emploi du passif permet donc de passer du constat d'événements externes subis par un personnage ( $c f .3 b)$ à l'expression de sentiments souvent défavorables pour tenter d'y mettre fin. Ainsi, l'expression des sentiments par le passif dote les élèves d'outils de communication émotive plus convaincants que le simple désaccord du fait qu'ils présentent le sentiment comme un changement d'état irrémédiable. Les sentiments peuvent être le moyen de réagir à une action subie mais aussi à une opinion.

\section{3d. Mise en perspective du point de vue d'un locuteur}

L'opinion ou l'affirmation d'un personnage ou de l'auteur d'un document peut également être mise en perspective de façon critique par l'énonciateur-apprenant à travers l'emploi de verbes introducteurs de discours rapporté qui lui permettent d'expliciter un point de vue discutable. Certains documents en contiennent d'ailleurs et peuvent permettre de les acquérir.

En (19), le verbe de discours rapporté claim est le moyen pour le personnage principal du roman 1984, de G. Orwell de remettre en question la version du Parti. Le narrateur rapporte les pensées de Winston, qui rapporte dans son journal intime les principes promulgués par une société arbitraire. Cette critique implicite mérite d'être explicitée par l'apprenant, qui peut, comme en 3a, faire varier l'origine modale en rapportant l'opinion de Winston :

(19)

The Party claimed, of course, to have liberated the proles from bondage. Before the Revolution they had been hideously oppressed by the capitalists, they had been starved and flogged, women had been forced to work in the coal mines (women still did work in the coal mines, as a matter of fact), children had been sold into the factories at the age of six. But simultaneously, true to the Principles of doublethink, the Party taught that the proles were natural inferiors who must be kept in subjection, like animals, by the application of a few simple rules. In reality very little was known about the proles. It was not necessary to know much. So long as they continued to work and breed, their other activities were without importance 
(...). Day and night the telescreens bruised your ears with statistics proving that people today had more food, more clothes, better houses, better recreations-that they lived longer, worked shorter hours, were bigger, healthier, stronger, happier, more intelligent, better educated, than the people of fifty years ago. Not a word of it could ever be proved or disproved. The Party claimed, for example, that today 40 percent of adult proles were literate: before the Revolution, it was said, the number had only been 15 per cent. The Party claimed that the infant mortality rate was now only 160 per thousand, whereas before the Revolution it had been 300 -and so it went on. It was like a single equation with two unknowns. It might very well be that literally every word in the history books, even the things that one accepted without question, was pure fantasy.

G. Orwell, 1984, Part I, section 7, Penguin, 1949

Les deux points de vue peuvent alors être explicitement opposés grâce à des mots de liaison exprimant le contraste. La manipulation du discours rapporté échappe alors au risque de l'exercice purement mécanique et formel par la variation des verbes introducteurs et des origines modales. Tant en reconnaissance qu'en production, le prétérit contribue à la remise en cause du point de vue du Parti par Winston, car il ne sert pas uniquement à marquer le passé, mais aussi à suggérer que l'opinion exprimée n'est plus pertinente au moment présent. Cela tend à montrer que la remise en question du point de vue implique plusieurs opérations différentes : une différenciation entre $\mathrm{S}_{1}$ et $\mathrm{S}_{0}$, mais aussi une rupture temporelle entre $\mathrm{T}_{1}$ et $\mathrm{T}_{0}$.

Le texte illustre en outre une variation intéressante entre complémentations personnelles en that à verbe conjugué et sujet différent, complémentation infinitive à sujet identique à celui du verbe de discours et incise impersonnelle. Suite à une pratique raisonnée de la langue sur les nuances véhiculées par ces constructions, l'enseignant pourrait demander à ses élèves d'illustrer cette variation lors d'une tâche prenant comme point de départ le discours d'un homme politique actuel dont les affirmations seraient remises en question.

En cycle terminal notamment, les tâches associées à la mise en perspective de points de vue différents sont la rédaction d'articles de journaux sur des questions données. Dans le but de fournir aux élèves des outils langagiers leur permettant d'accomplir ces tâches, on remarque que $\mathrm{H}$. Wyld (2001: 146-150) offre un classement intéressant des verbes introducteurs de pensée rapportée ou de discours rapporté en fonction de paramètres simples: soit le verbe implique un effacement maximal de l'énonciateur (colonne de droite), soit une concordance (colonne du milieu), soit une discordance (colonne de gauche) entre la source modale $\mathrm{S}_{1}$ citée et l'énonciateur $\mathrm{S}_{0}{ }^{9}$. Ce classement pourrait aboutir en classe d'anglais à la distribution d'une tool box sous la forme du tableau ci-dessous :

Tableau 2

\begin{tabular}{|l|l|l|}
\hline Agreement & Disagreement & Neutrality \\
\hline acknowledge, admit, agree, know, understand & believe, claim, be convinced, forget, imagine, & say, think \\
\hline
\end{tabular}

Les énoncés construits à partir de ces verbes introducteurs permettraient aux élèves d'expliciter le point de vue d'un personnage par rapport à un autre ou de le déclarer comme discutable. 
Les nouveaux programmes de lycée ${ }^{10}$ associent désormais clairement la traduction à l'activité en langue étrangère :

« À l'articulation des activités langagières : la médiation

La médiation introduite dans le CECRL consiste à expliciter un discours lu et entendu à quelqu'un qui ne peut le comprendre. En termes scolaires, elle se traduit en une série d'exercices qui vont de la paraphrase à la traduction.

À l'oral comme à l'écrit, l'élève médiateur :

- prend des notes, paraphrase ou synthétise un propos ou un dossier documentaire pour autrui, par exemple à l'intention de ses camarades en classe ;

- identifie les repères culturels inaccessibles à autrui et les lui rend compréhensibles ;

- traduit un texte écrit, interprète un texte oral ou double une scène de film pour autrui ;

- anime un travail collectif, facilite la coopération, contribue à des échanges interculturels, etc. »

(Nouveaux programmes de Seconde, $2018: 10$ )

L'analyse du discours de Winston et de ses variations pourrait déboucher sur la justification de solutions de traduction des verbes introducteurs de discours. En effet, la mise en perspective de la source modale de l'énoncé est une problématique également présente en traduction, par exemple celle des verbes d'opinion think et believe vers le français. F. Doro-Mégy (2008: 31 \& 186) indique d'ailleurs qu'un des critères de traduction de think par croire ou s'imaginer au détriment de penser est précisément l'«altérité radicale», ou point de vue contradictoire entre le sujet énonciateur et le sujet de l'énoncé. H. Chuquet \& R. Nita (2013) dégagent également des critères pour distinguer les formes françaises d'après moi, selon moi, pour moi, à mon avis pour traduire say et think.

\section{3e. Médiation et autonomie}

La détermination du point de vue est en tout cas une compétence transférable permettant à terme l'autonomie des élèves. Dans le but d'atteindre cette autonomie, les nouveaux programmes de Seconde, Première et Terminale $(2018: 10)^{11}$ explicitent la compétence de médiation. Cette compétence invite l'enseignant à une pratique de classe explicite qui vise l'autonomie : devant les élèves, puis avec les élèves, puis laisser faire les élèves, en groupe ou binôme puis finalement seuls. Tout en visant l'autonomie, la pratique de classe vise également à pallier l'hétérogénéité. Celle-ci nécessite que les élèves plus autonomes explicitent des opinions implicites à des élèves moins autonomes. L'explicitation est donc une activité conjointe de production et de compréhension réunissant à la fois un énonciateur-rapporteur et ses camarades, dans le but d'une régulation, ou mise à niveau des connaissances et de la compréhension.

Cette explicitation peut concerner le point de vue d'un personnage ou auteur: le tableau synthétique des descripteurs de la médiation $(2018: 22)^{12}$ au niveau B2 indique que l'apprenant "peut (en langue $\mathrm{Y}$ ), faire une synthèse et rendre compte d'informations et d'arguments venant de diverses sources orales et écrites (en langue $\mathrm{X}$ ) ; peut comparer, opposer et synthétiser (en langue Y), des informations et points de vue différents (en langue $\mathrm{X}$ ) ».

Les mêmes outils pourraient être utilisés pour étudier le texte (20) dont le contenu correspond à l'entrée de l'Art de vivre ensemble niveau Seconde, car il aborde les décalages entre générations. En compréhension, le point de vue du jeune apprenant ne 
peut être que différent de celui de Jolyon dans le passage ci-dessous, où il ressort comme un vieil avare espérant toujours faire de l'argent après sa mort. Il entretient en outre des relations conflictuelles avec son gendre du fait de sa relation fusionnelle avec sa fille :

(20)

At five o'clock the following day old Jolyon sat alone, a cigar between his lips, and on a table by his side a cup of tea. He was tired, and before he had finished his cigar he fell asleep. A fly settled on his hair, his breathing sounded heavy in the drowsy silence, his upper lip under the white moustache puffed in and out. From between the fingers of his veined and wrinkled hand the cigar, dropping on the empty hearth, burned itself out.

The gloomy little study, with windows of stained glass to exclude the view, was full of dark green velvet and heavily-carved mahogany - a suite of which old Jolyon was wont to say: 'Shouldn't wonder if it made a big price some day!'

It was pleasant to think that in the afterlife he could get more for things than he had given.

In the rich brown atmosphere peculiar to back rooms in the mansion of a Forsyte, the Rembrandtesque effect of his great head, with its white hair, against the cushion of his high-backed seat, was spoiled by the moustache, which imparted a somewhat military look to his face. An old clock that had been with him since before his marriage forty years ago kept with its ticking a jealous record of the seconds slipping away forever from its old master. [...]

He woke. June had gone! James had said he would be lonely. James had always been a poor thing. He recollected with satisfaction that he had bought that house over James's head.

Serve him right for sticking at the price; the only thing the fellow thought of was money. Had he given too much, though? It wanted a lot of doing to - He dared say he would want all his money before he had done with this affair of June's. He ought never to have allowed the engagement.

John Galsworthy, The Man of Property (1906)

Les deux derniers paragraphes en particulier, illustrant le discours indirect libre, se prêtent à l'explicitation par les apprenants du caractère exigeant de Jolyon par rapport à sa fille, mais aussi et surtout son gendre qu'il n'estime pas beaucoup. En production, la manipulation du discours rapporté peut être transférée vers des tâches visant à donner la parole à la fille et au gendre de Jolyon, mais aussi à récupérer l'héritage de Jolyon tout en offrant l'occasion de malentendus entre membres de la famille. Cette tâche implique elle aussi une forme de régulation qui pourrait prendre la forme d'un compromis.

\section{Conclusion}

$\mathrm{Au}$ final, expliciter les relations intersubjectives implique de partir d'un état implicite de ces relations au niveau énonciatif, qu'elles soient non présentes dans l'original de la traduction, dissimulées derrière des contraintes objectives, ou seulement suggérées par un énonciateur dans un dialogue. Elles sont finalement explicitées par le traducteur pour le lecteur de la traduction, par l'interlocuteur, dans son intérêt, au fil du temps en diachronie ou par les élèves dans un contexte didactique. Ce que l'on tente d'expliciter est souvent la véritable intention ou opinion du premier locuteur. L'explicitation est donc une activité conjointe et dissymétrique de production et de reconnaissance réunissant un premier locuteur et un deuxième qui reconstruit et réévalue un premier sens produit. Il ressort que l'explicitation de l'intersubjectivité est un enjeu de pouvoir 
portant sur des demandes implicites qui nous sont adressées, des rapports de forces entre personnes ou personnages, des conflits ou des différences d'opinion.

Cette explicitation se matérialise à un niveau prédicatif par l'emploi de verbes transitifs, notamment au passif. Leurs arguments renvoient à des animés humains distincts. Cet emploi de verbes transitifs reflète globalement le développement de repérages subjectifs au détriment des repérages spatio-temporels. Ces repérages, qui constituent l'opération fondamentale sur laquelle repose la TOE, interviennent aussi bien dans la construction du sens, en expression que dans la reconstruction du sens en compréhension ou traduction.

Cette explicitation se vérifie dans des domaines différents (traduction, diachronie, didactique), des faits de langue différents (adverbes de manière, verbes directifs, verbes d'opinion) correspondant à des types de modalité eux aussi différents (assertion rapportée, modalité appréciative, contrainte intersubjective).

Toutefois, les faits de langue à expliciter se situent plutôt dans le cadre du discours direct pour la diachronie, alors qu'ils se situent surtout dans le discours rapporté en traduction et en didactique. Pour tenter d'accéder au sens, on essaie dans tous les cas de l'expliciter par des formes subjectives et intersubjectives, notamment le passif qui véhicule une appréciation subjective. On remarque toutefois que want et need sont réfractaires au passif malgré leur subjectivisation, du fait des contraintes de leur complémentation.

\section{BIBLIOGRAPHY}

Bay, André (1958 [1980]). Alice au pays des merveilles. Paris, Librairie Générale Française.

Bué, Henri (1869 [1972]). Aventures d'Alice au pays des merveilles. New York, Dover.

Caffi, C., Janney, R. W. (1994). « Toward a pragmatics of emotive communication », Journal of Pragmatics, 22 (3-4), pp. 325-373. URL: https://doi.org/10.1016/0378-2166(94)90115-5

Chuquet, H., Nita, R. (2013). «Manifestations incidentes du point de vue : quelques marqueurs du français et leurs équivalents en anglais ", in H. Chuquet, R. Nita, \& F. Valetopoulos (éds) Des sentiments au point de vue : études de linguistique contrastive, pp. 211-228. Presses Universitaires de Rennes.

Culioli, A. (1990). Pour une Linguistique de l'énonciation, Opérations et représentations, Tome 1. Collection HDL, Paris : Ophrys.

Culioli, A. (1999). Pour une Linguistique de l'énonciation, Formalisation et opérations de repérage, Tome 2. Collection HDL, Paris : Ophrys.

Deschamps, A. (2006a). « Les verbes de parole : spécificités et formes schématiques », in C. Normand \& D. Ducard (éds) Antoine Culioli, Un homme dans le langage. Actes du colloque de Cerisy en l'honneur d'Antoine Culioli, collection HDL, pp. 267-284. Paris : Ophrys. 
Deschamps, A. (2006b). « Pour un traitement formel énonciatif des compléments de verbe à forme non-finie ", in C. Delmas (éd) Complétude, cognition, construction linguistique, pp. 135-145. Presses de la Sorbonne Nouvelle. URL: https://books.openedition.org/psn/3146?lang=fr Doro-Mégy, F. (2008). Etude croisée de think, believe, croire et penser, Linguistique comparée et traduction, Numéro Spécial. Paris : Ophrys.

Gilbert, E. (1993). « La théorie des opérations énonciatives d'Antoine Culioli », in P. Cotte (éd) Les théories de la grammaire anglaise en France, pp. 63-96. Paris : Hachette.

Groussier, M.-L. (1993). « Où est le sens ? Ou l'impossible feinte interénonciative », in Cahiers Charles V, Travaux de linguistique énonciative, $\mathrm{n}^{\circ} 16$, Le sens, pp. 17-39. Institut d'anglais et Université Paris VII. URL: https://www.persee.fr/doc/cchav_0184-1025_1993_num_16_1_1085

Groussier, M.-L. (2000). « Subjectivisation croissante de la valeur des modaux au cours de l'histoire de l'anglais ", in J. Pauchard (éd) La modalité et les modaux en diachronie et synchronie (domaine anglais), Actes des journées scientifiques 1998/1999, pp. 73-101. Presses Universitaires de Reims.

Groussier, M.-L. (2002). « Evolution des indicateurs de modalité déontique et occultation de la subjectivité ». Journée ALAES sur la modalité. URL: https://alaesfrance.files.wordpress.com/ 2015/04/groussier2002.pdf

Guillemin-Flescher, J. (1981). Syntaxe comparée du français et de l'anglais. Paris : Ophrys.

Lapalme, Pierre (1980). Alice au pays des merveilles. Neuilly-sur-Seine, Dargaud Jeunesse.

Lattès (1987). Alice au pays des merveilles. Bibliothèque Lattès.

Plantin, C. et al. (2000). Les émotions dans les interactions. Presses Universitaires de Lyon.

Le Bot M.-C., et al. (2008). Pragmatique de la reformulation. Types de discours. Interactions didactiques. Presses universitaires de Rennes.

Marcireau, Jacques [sic] (1983). Alice au pays des merveilles. Paris, Metro Editions Internationales. Merle, Magali (1990). Les Aventures d'Alice au pays des merveilles. Les Langues Modernes / Bilingue, Livre de Poche, Librairie Générale Française.

Parisot, Henri, (1968). Alice au pays des merveilles. Paris, Flammarion.

Peter, Charlotte (1971). Alice au pays des merveilles. Zurich, Silva.

Polge, O. (2013). «Les adverbes d'état subjectif dans Alice's Adventures in Wonderland et leurs traductions ", in H. Chuquet, R. Nita, \& F. Valetopoulos (éds) Des sentiments au point de vue : études de linguistique contrastive, pp. 155-174. Presses Universitaires de Rennes.

Polge, O. (2015). «I want you to and I need you to in Modern and Present-Day English », in J.-R. Zamorano-Mansilla, C. Maíz, E. Domínguez \& Mª Victoria Martín de la Rosa (éds) Thinking modally: English and Contrastive Studies on Modality, pp. 23-45. Cambridge Scholars.

Polge, O. (2017). « Sentiments et intersubjectivité en classe d'anglais », La Clé des Langues (Lyon: ENS LYON/DGESCO). ISSN 2107-7029. URL: http://cle.ens-lyon.fr/linguistique/sentiments-etintersubjectivite-en-classe-d-anglais-337372.kjsp

Prophétie, Robert \& Prophétie André (1949). Alice au pays des merveilles. Paris, Editions G. P.

Quirk, R. et al. (1985). A Comprehensive Grammar of English. London: Longman.

Rouard, Philippe (1984). Alice au pays des merveilles. Paris, Hachette.

Scott, Olive Ray \& Keukles Ed (1937). Aventures d'Alice au pays des merveilles. Paris, Hachette. 
Simonin, O. (2015). « Propos liminaire sur la modalisation a posteriori et le commentaire », in Anglophonia [Online], 19 | 2015, Online since 15 July 2015, connection on 24 June 2020.

URL:http://journals.openedition.org/anglophonia/456 ; DOI : https://doi.org/10.4000/ anglophonia.456

Traugott, E.C. (1995). "Subjectification in grammaticalisation", in Dieter Stein \& Susan Wright (éds) Subjectivity and Subjectivisation, pp. 31-54. Cambridge: Cambridge University Press.

Traugott, E.C. (2010). “(Inter)subjectivity and (inter)subjectification: a reassessment”, in Kristin Davidse, Lieven Vandelanotte, Hubert Cuyckens (éds), Subjectification, Intersubjectification and Grammaticalization, pp. 29-74. Walter de Gruyter.

Vinay, J.-P., Darbelnet, J. (1958/1967). Stylistique comparée du français et de l'anglais. Paris : Didier.

Visser, F.T. (1963-73). An Historical Syntax of the English Language, I-IV. Leiden: E.J. Brill.

Wyld, H. (2001). Subordination et énonciation, in Cahiers de recherche en grammaire anglaise, Numéro spécial, Paris, Ophrys.

\section{NOTES}

1. Dubois, J. \& F. Dubois-Charlier (2005). Adjectifs en français, Aix, 303 pages.

2. Polge, O. (2013). «Les adverbes d'état subjectif dans Alice's Adventures in Wonderland et leurs traductions ", in Chuquet, H. Nita, R. \& Valetopoulos, F. (éds), Des sentiments au point de vue : études de linguistique contrastive, Presses Universitaires de Rennes, pp. 155-174.

3. Dubois-Charlier, F., Vautherin, B. (1997). Syntaxe anglaise: examens et concours de l'enseignement supérieur. Vuibert Supérieur.

4. Polge, O. (2007). Etude du prédicat want et de ses constructions en anglais contemporain. Thèse de doctorat, Université Paris Diderot, 556 pages.

5. Je remercie Laetitia Léonarduzzi, Sophie Dufossé et mes autres relecteurs pour leurs remarques et critiques.

6. Traduction personnelle : (12a) Lazarotto: Un chancre de méfait hante mon giron, plus lourd qu'il ne puisse porter. Une sage femme m'aiderait à m'en soulager. Lorenzo : Cette sage femme donc je serai, pour te défaire prestement de ce piètre farfadet qui te grève et te tourmente.

7. L'expression d'une contrainte intersubjective suppose au niveau de la construction du prédicat la mise en place d'une concaténation entre deux relations prédicatives, qui implique le schéma général suivant:

$$
\ll \text { A R B }>\geqslant<\text { (a) } \mathrm{rb}>>
$$

Dans ce schéma, l'enchâssée < a r b > est repérée par rapport à l'enchâssante < A R B > (cf. A. Deschamps (2006b)). Dans l'enchâssante, le terme A, premier argument, désigne l'origine subjective de la contrainte, qui constitue un repère et le terme $B$, deuxième argument, constitue la cible de la contrainte et correspond au N2 mentionné. L'enchâssée $<$ (a) $r b>$ désigne l'action visée par la source de la contrainte.

8. Malgré son étude en partie 2, need n'a pas été intégré dans cette liste puisque son emploi de contrainte intersubjective est encore récent et peu évident. En tout cas, force ou make sont passivables lorsqu'ils sont suivis d'un complément propositionnel infinitif, à la différence de need et want.

9. Même si ce classement rappelle la distinction tripartite entre verbes factifs/contrefactifs/non factifs émise par Kiparsky \& Kiparsky (1969), l'approche de H. Wyld dans le cadre de la TOE repose sur le fait qu'un verbe n'est pas factif, contrefactif ou non factif en soi mais que ses sens découlent de ses conditions d'emploi au niveau énonciatif (temps, modalité, aspect, personne, 
négation, voix). Par exemple, un verbe tel que think peut tout à fait apparaître dans plusieurs colonnes de ce tableau. Suite à cette étude, on peut rajouter aux facteurs énonciatifs la dimension intersubjective de l'énoncé, dont les différentes prises en charge priment sur sa valeur de vérité.

10. https://cache.media.education.gouv.fr/file/SP1-MEN-22-1-2019/95/2/ spe585_annexe1_1062952.pdf

11. https://cache.media.education.gouv.fr/file/SP1-MEN-22-1-2019/95/2/ spe585_annexe1_1062952.pdf

12. https://cache.media.education.gouv.fr/file/SP1-MEN-22-1-2019/70/3/ spe585_annexe2CORR_1063703.pdf

\section{ABSTRACTS}

Whether a given utterance pertains to reported assertion, qualifying modality, or intersubjective constraint, the interaction it involves between two animate subjects is often made explicit in different domains, among others the presence of a reporting verb in present-day English, the more and more explicit meaning of volitional verbs in historical English, or again the translation into French of manner adverbs as past participles conveying a qualifying modality stemming from the subject of the utterance. In those various fields, intersubjectivity often involves the use of a transitive verb to express a change of state, with a view to greater efficiency within the struggle for power between speaker and co-speaker, who strive to make their adressee act as they please. The language fields tackled in translation and historical English will lead up to their practical use in an English class in the last year of French high school, which involves accessing the implicit meaning of documents, through guiding lines such as « private and public spaces » or « art and power ». Written comprehension requires making the characters' points of view, their implicit requests and conflicts more explicit. The modern language class is therefore a place where interactions are brought out or carried out thanks to the use of language means. Although the processes at work are similar, intersubjectivity is to be found predominantly within direct speech for historical English, whereas in translation and didactics, it is made explicit out of texts illustrating either direct speech or past events.

Malgré des types de modalité différents (assertion rapportée, modalité appréciative, contrainte intersubjective), l'intersubjectivité, ou interaction entre deux sujets animés humains dans un énoncé est souvent explicitée par un verbe transitif, dans des domaines différents : choix du verbe introducteur de discours rapporté en synchronie, explicitation du sens de verbes de volition en diachronie, traduction des adverbes de manière par des participes passés exprimant une modalité appréciative émanant du sujet de l'énoncé. Dans ces domaines différents, l'intersubjectivité implique souvent l'explicitation d'un changement d'état par un verbe transitif, dans le but d'une plus grande efficacité dans le cadre du conflit de pouvoir qui consiste pour le locuteur à faire agir son interlocuteur selon son choix. Les faits de langue abordés en traduction et diachronie déboucheront sur leur emploi pratique en classe d'anglais : le cycle terminal, qui requiert l'accès à l'implicite des documents, contient les axes « Espace privé et espace public » ou encore «Art et pouvoir ». La compréhension écrite nécessite l'explicitation des points de vue des personnages, de demandes implicites et de conflits entre parties. Un cours de langue vivante est donc un lieu où des interactions sont dégagées en compréhension, ou engagées entre élèves grâce aux moyens langagiers manipulés. Même si les processus à l'œuvre sont similaires, 
l'intersubjectivité se situe donc plutôt dans le cadre du discours direct en diachronie, alors qu'en traduction et en didactique, l'explicitation de l'intersubjectivité se fait à propos d'un texte soit au discours direct, soit relatant des événements passés.

INDEX

Mots-clés: explicitation, intersubjectivité, assertion rapportée, modalité appréciative, contrainte intersubjective

Keywords: explicitation, intersubjectivity, reported assertion, qualifying modality, intersubjective constraint

\section{AUTHOR}

OLIVIER POLGE

Université de Limoges, CeReS EA3648 